\title{
Wie soll man begutachten?
}

\section{Alexander Kiss}

Prof. Dr. med., Mitglied der FMH

Menschen mit körperlichen Beschwerden ohne organische Ursache werden in der Schweiz in Bezug auf einen Rentenanspruch sehr unterschiedlich begutachtet. Eine Zeitlang bestand eine Art Laissez-faire mit vielen Berentungen, die Milliarden Franken kosteten. Danach folgte ein restriktives Vorgehen, bestimmte Diagnosen wurden von einer Berentung prinzipiell ausgeschossen (Pathogenetisch Ätiologisch Unklare Syndromale Beschwerdebilder Ohne Nachweisbare Organische Grundlage PÄUSBONOG). Das BGE-Urteil 9C_492/2014 vom 03.06.2015 soll die bisherige Praxis ersetzen. Nicht die Diagnose, sondern der Nachweis der Behinderung ist im Fokus der Begutachtung, der mit Hilfe von Indikatoren erfolgt. Ob dieser Paradigmenwechsel in der Praxis von Gutachtern, die die funktionelle Leistungsfähigkeit des Versicherten beurteilen und somit die Entscheidungsgrundlage für die Versicherungen und gegebenenfalls für Gerichte liefern, vollzogen oder unterlaufen wird, ist fraglich [1].

In der alltäglichen Praxis ist - trotz gegenteiliger Beteuerungen - der totgesagte Leib-Seele-Dualismus recht lebendig. Bei somatischen Beschwerden mit organisch nachweisbarer Ursache gibt es eher eine Rente, ohne organisch nachweisbare Ursache gibt es eher keine Rente. Manche Beschwerdebilder sind jedoch nicht so eindeutig zuzuordnen und Gutachter sind dann geneigt, vorschnell «keine organische Ursache» und keine Einschränkung der Arbeitsfähigkeit festzustellen.

\section{CRF und Arbeitsfähigkeit}

Ein Beispiel für ein solches nicht gut zuordenbares Beschwerdebild war das Cancer-related Fatigue (CRF) bis zum Urteil des Bundesgericht vom 13.06.2013. Das Gericht stellte damals neu fest: Als Begleitsymptom

\section{Résumé}

L'article montre, en prenant l'exemple de la fatigue liée au cancer (CRF), que tous les tableaux cliniques ne sont pas clairement attribuables en médecine. Dans de tels cas, les experts ne devraient pas conclure hâtivement: "pas de cause organique, pas de rente». L'arrêt 9C_492/2014 du 03.06.15 du Tribunal fédéral va dans le bon sens à cet égard. L'expertise doit se concentrer sur la preuve du déficit fonctionnel (et non sur le diagnostic) et l'établir à l'aide d'indicateurs. II n'est pas sûr que ce changement de paradigme soit effectivement appliqué sur le fond dans la pratique. von Krebserkrankungen und ihrer Therapie liege der CRF aber «zumindest mittelbar eine organische Ursache zu Grunde» und es sei deshalb nicht zu rechtfertigen, bei CRF die gleichen Kriterien anzuwenden, wie sie bei somatoformen Schmerzstörungen gelten.

In den letzten 20 Jahren habe ich viele Patienten mit CRF bezüglich ihrer Arbeitsfähigkeit begutachtet und im Aktenstudium Einsicht gewonnen, welche Strategien Gutachter und IV-Stellen gebrauchen, um die Arbeitsfähikeit bei Beschwerdebildern zu beurteilen, die ihnen oft wenig vertraut sind und deren organische Ursachen unklar sind. Die einzelnen Strategien der Gutachter zur Beurteilung der Arbeitsfähigkeit scheinen mir berichtenswert, weil auch in Zukunft bei «unklaren» und/oder dem Begutachter wenig vertrauten Beschwerdebildern ähnliche Strategien angewendet werden könnten, meist zum Nachteil des zu Begutachtenden.

Tabelle 1: Proposed (1998 draft) ICD-10 Criteria for CancerRelated Fatigue [12].

Six (or more) of the following symptoms have been present every day or nearly every day during the same 2-week period in the past month, and at least one of the symptoms is (A1) significant fatigue.

Significant fatigue, diminished energy, or increased A1 need to rest, disproportionate to any recent change in activity level

A2 Complaints of generalized weakness or limb heaviness

A3 Diminished concentration or attention

Decreased motivation or interest to engage in usual activities

A5 Insomnia or hypersomnia

A6 Experience of sleep as unrefreshing or nonrestorative

A7 Perceived need to struggle to overcome inactivity

A8 Marked emotional reactivity (e.g., sadness, frustration, A8 or irritability) to feeling fatigued

A9 Difficulty completing daily tasks attributed to feeling fatigued

A10 Perceived problems with short-term memory

A11 Postexertional malaise lasting several hours

The symptoms cause clinically significant distress or

B impairment in social, occupational, or other important areas of functioning.

There is evidence from the history, physical examina-

C tion, or laboratory findings that the symptoms are a consequence of cancer or cancer therapy.

The symptoms are not primarily a consequence of comorbid psychiatric disorders such as major depression, somatization disorder, somatoform disorder, or delirium. 
Was ist das CRF? Während und nach einer Krebserkrankung sind Müdigkeit und rasche Erschöpfbarkeit häufig. Die Intensität und die Dauer der Symptome sind individuell sehr unterschiedlich. Nur bei einem kleinen Teil der Menschen sind die Beschwerden nach einer Krebserkrankung so ausgeprägt, dass die Arbeitsfähigkeit trotz Rehabilitation eingeschränkt bleibt. Die Diagnosestellung ist deskriptiv, die klinischen Kriterien sind in Tabelle 1 ersichtlich.

Zum Ausschluss von somatischen Ursachen der Müdigkeit bei Patienten nach einer Krebserkrankung (z.B. Anämie, Hypothyreose etc.) bestehen Algorithmen [2]. Die genauen Ursachen des CRF sind nicht bekannt. Vermutet wird eine Interaktion von somatischen Faktoren (z.B. Tumorart, Art der Behandlung, Cytokine etc.), psychischen (z.B. Depression) und sozialen Faktoren.

Zur Therapie des CRF werden dosiertes körperliches Training, Psychoedukation / kognitive Verhaltenstherapie, Medikamente und/oder Komplementärmedizin empfohlen [3, 4]. Der Erfolg der therapeutischen Massnahmen ist bescheiden.

\section{Welche Strategien verwenden Gutachter zur Beurteilung des CRF und der damit verbundenen Arbeitsfähigkeit?}

Im Folgenden beschreibe ich zuerst die Strategie des Gutachters, zitiere dann aus seinem oder ihrem Gutachten und kommentiere am Ende. Manche Gutachter kombinieren mehrere Strategien, um ihre Einschätzung zur Arbeitsfähigkeit zu begründen.

\section{Strategie 1: Zuordnung zu PÄUSBONOG (vor dem BGE-Urteil 9C_492/2014 vom 03.06.2015)}

\section{Zitat aus dem Gutachten:}

"[Bei der] Diagnose «Cancer related Fatigue» handelt es sich um eine Diagnose, die aus versicherungsmedizinischer Sicht der Gruppe von Chronic-Fatigue-Syndrome, Fibromyalgie und verwandten Störungen zuzuordnen ist.»

«Was die neueste BG-Rechtsprechung betrifft, kommt ein weiterer Aspekt hinzu: die Diagnose eines ChronicFatigue-Syndroms wird heute bei mangelnder Komorbidität der Gruppe der syndromalen Beschwerden zugeordnet, welchen eine IV-Relevanz abgesprochen wird.»

\section{Kommentar:}

Das «Cancer-related», die ursächliche Beziehung der Krebserkrankung zur Müdigkeit, wird nicht berücksichtigt und die Beschwerden werden zum Chronic-
Fatigue-Syndrom. Damit besteht keine «IV-Relevanz», d.h. keine eingeschränkte Arbeitsfähigkeit. Das Urteil des Bundesgericht vom 13.06.2013 verunmöglicht diese Strategie.

\section{Strategie 2: Beschwerden sind glaubhaft, aber dem Gutachter fehlt eine passende Diagnose.}

\section{Zitat aus Gutachten:}

«Die Müdigkeit ist glaubhaft, aber die Beschwerden korrelieren nicht mit einem objektiven Befund oder einem organischen Korrelat.»

\section{Kommentar:}

Die Müdigkeit ist für den Gutachter zwar glaubhaft, aber ihm fehlen die diagnostischen Kriterien für Cancer-related Fatigue, um diese glaubhaften Beschwerden einzuordnen. Die Konsequenz für den glaubwürdigen Betroffenen: keine eingeschränkte Arbeitsfähigkeit.

\section{Strategie 3: Passt eine Diagnose aus meinem Fach zu den Symptomen?}

Der Gutachter ist Psychiater: Manche versuchen die Symptome des Patienten aus ihrem Fach zu erklären. Es besteht die Gefahr, dass die Symptome des CRF als depressive Symptome beschrieben werden und dadurch die Fehldiagnose «Depression" gestellt wird, oder dass bei einer Komorbidität von CRF und Depression nur die Diagnose Depression gestellt wird.

\section{Kommentar:}

Bei Patienten mit CRF ist die Gabe von Antidepressiva nachweislich wirkungslos [5]. Bei Patienten mit CRF und Depression kommt es durch die Gabe von Antidepressiva möglicherweise zu einer Verbesserung der depressiven Symptome, nicht aber der Symptome des CRF. Die Beurteilung der Arbeitsfähigkeit hängt allein von der vermeintlichen Schwere und Behandelbarkeit der depressiven Symptome ab. Das CRF bleibt unberücksichtigt, weil nicht diagnostiziert.

Der Gutachter ist Onkologe: Manche sind versucht, das Beschwerdebild «organisch» zu erklären:

\section{Zitat aus dem Gutachten:}

«Onkologischerseits ist unserer Ansicht nach von einer dauerhaft eingeschränkten Arbeitsfähigkeit von 50\% auszugehen. Dies resultiert aus der eingeschränkten kardiopulmonalen Situation und der daraus auch reduzierten allgemeinen und geistigen Leistungsfähigkeit (Konzentration/Aufmerksamkeit). Gegebenenfalls kann dies durch weitere Funktionsuntersuchungen objektiviert werden.» 


\section{Kommentar:}

In den weiterführenden Untersuchungen ist nichts objektivierbar. Die fehlende «eingeschränkte kardiopulmonale Situation» macht für den Onkologen die Beurteilung der Arbeitsfähigkeit zum Problem.

\section{Strategie 4: Angebliches Zitieren aus der wissen- schaftlichen Literatur ohne Referenz}

\section{Zitat aus dem Gutachten:}

«Es besteht eine ausgesprochene Unspezifität der Symptome, welche zudem auch nicht sensitiv sind. Dementsprechend gibt es bisher auch keine anerkannten Langzeitstudien, die nachweisen können, dass mehrere Monate bis mehrere Jahre nach Manifestation eines behandelten Karzinoms mögliche Symptome sich relevant von der Durchschnittsbevölkerung abheben.»

\section{Kommentar:}

In mehreren Studien wurde nachgewiesen, dass Krebspatienten im Langzeitverlauf häufiger und ausgeprägter Symptome von Fatigue haben als die Normalbevölkerung [6-8]. Bezüglich Arbeitsfähigkeit steht in einer 2012 veröffentlichten Review: «Depending on the course and severity of the underlying disease, the effects of CRF can range from temporary indisposition to inadequate coping with everyday life and social withdrawal all the way to the inability to perform one's job and earn a living, leading to economic hardship for the patient and an additional economic burden for society at large» [9].

Strategie 5: «keine objektiven Befunde mehr, welche noch eine funktionelle Einschränkung auf die Leistungsfähigkeit begründen würden.»

\section{Zitat aus einem Brief der IV:}

«Frau X ist seitdem in vollständiger klinischer Remission und objektiv anhaltend beschwerdefrei. Eine 'Cancer' assoziierte, anhaltende Müdigkeit ist deshalb nicht mehr nachvollziehbar. [...] Es bleibt nicht nachvollziehbar, warum die im Jahre XX durchgeführte Stammzelltransplantation eine 'chronisch reduzierte Leistungseinschränkung' bewirken sollte, es finden sich heute keine objektiven Befunde mehr, welche noch eine funktionelle Einschränkung auf die Leistungsfähigkeit begründen würden.»

\section{Zitat aus der wissenschaftlichen Literatur (statt eines Kommentars):}

«33\% of cancer survivors experience persistent fatigue of unknown origin, causing significant impairment in quality of life. Despite the prevalence and impact of this symptom, currently, there are no empirically validated treatments for persistent cancer-related fatigue» [10].

\section{Strategie 6: Kombination von Fehldiagnose, Unwissen und Entwertung des betreuenden Arztes}

Der behandelnde Psychiater stellt bei seiner 39-jährigen Patientin mit einem metastasierenden Brustkrebs ein CRF mit einer Einschränkung der Arbeitsfähigkeit fest. Die Patientin wird begutachtet. Das Gutachten stellt fest, dass die Patientin kein Cancer-related Fatigue, sondern eine Neurasthenie hat und somit 100\% arbeitsfähig ist. Die Patientin ficht den Entscheid an.

\section{Aus der Stellungnahme des Gutachterinstitutes:} Der Psychiater schreibt: «[...] erachte es als wenig sinnvoll, hier eine Expertendiskussion oder einen Fachdisput über die Differentialdiagnose Neurasthenie/Mammacarcinom versus cancer-related fatigue zu führen. Entscheidend ist der Einfluss auf die Arbeitsfähigkeit. Hierzu sei bemerkt, dass - obschon die Vt subjektiv über Müdigkeit, Energielosigkeit und Erschöpfung klagt - gemäss objektiven Befunden Mimik und Gestik normal waren, ebenso der Antrieb, die Stimmung ausgeglichen, die Aufmerksamkeit und Auffassung normal, die Selbstwertregulation gut [...]"

Der Internist schreibt: «Aus internistischer Sicht ist anzumerken, dass interessanterweise der Psychiater Dr. X sich über das 'CRF' äussert, also einer Müdigkeit, die einer somatischen Erkrankung bzw. einer ehemaligen somatischen Erkrankung und Therapie zugeordnet werden soll.»

\section{Aus meiner Exploration der Patientin:}

«Nach dem Aufstehen müsse sie ungefähr eine halbe Stunde sitzen, dann könne sie duschen und das Frühstück einnehmen. Am Vormittag sei maximal das Betten-Abdecken bzw. eine Waschmaschine möglich, dann sei ihre Energie erschöpft. Nach dem Mittagessen gehe sie mit dem Hund eine halbe Stunde langsam spazieren, und damit sei der Tag schon gelaufen. Oft müsse sie sich den ganzen Nachmittag ausruhen. Ein Arztbesuch bzw. eine Kommission sei oft sehr anstrengend und oft zu viel. Der Schlaf sei schlecht und sie wache oft auf in der Früh, ohne wirklich eine Erholung und mehr Energie durch den Schlaf zu haben.

Wenn sie sich dazu zwingen würde, mehr zu tun als sie eigentlich kann, wie zum Beispiel zwei Termine wahrzunehmen, dann würde sie massiv dafür bezahlen, indem sie in den nächsten ein bis zwei Tagen vollkommen ohne Energie sei.

Schwierig ist auch im Sozialleben, dass sie nicht weiss, wie viel Energie sie zu einem vereinbarten Termin 
haben wird, so dass sie einen Termin bzw. Besuch auch kurzfristig absagen muss. Diese bleierne Müdigkeit habe zu einer radikalen Änderung ihres Alltags geführt:

Den Haushalt kann sie alleine selbst nicht mehr versorgen, sie hat eine Putzfrau; sie geht nicht mehr alleine einkaufen, sondern der Mann geht mit, auch um zu tragen; sie versorgt den Garten nicht mehr, was sie früher gewohnt war, und die Kinder haben einige Funktionen übernommen, die sie nicht mehr ausfüllen kann.»

Die Kriterien für Cancer-related Fatigue sind erfüllt und die Patientin hat eine erheblich eingeschränkte Arbeitsfähigkeit.

\section{Der Gutachter ist unwissend:}

Zum Zeitpunkt des Gutachtens könnten die Leitlinien zur sozialmedizinischen Begutachtung der Leistungsfähigkeit bei Mamma-Karzinom der Deutschen Rentenversicherung vom 18.11.2011 bekannt sein [11]: «Verstanden wird unter CRF ein krankheitswertiges, unüberwindliches, anhaltendes, ganzkörperliches $\mathrm{Ge}$ fühl, das gekennzeichnet ist durch eine verminderte Kapazität für körperliche und geistige Betätigung [...]. In der Literatur finden sich für das CRF hohe Prävalenzzahlen. Unterschieden wird zwischen einem akuten und einem chronischen Syndrom. Das akute CRF tritt passager und in der Regel therapiebegleitend auf und bildet sich nach einer ausreichenden Zeit der Rekonvaleszenz zurück. Beim seltener vorkommenden chronischen CRF persistieren die Symptome länger als sechs Monate nach Ende der Therapie» (S. 22/23).

\section{Die Gutachter entwerten den betreuenden Arzt:}

Die Differentialdiagnose des betreuenden Arztes wird als sinnlos dargestellt und dem Psychiater wird das Recht abgesprochen, sich zu dem somatischen Leiden seiner Patientin und den Folgen zu äussern.

Die vorgestellten Strategien zur Begutachtung von CRF basieren auf Unwissen, Desinteresse an wissenschaftlicher Literatur und der Entwertung des betreuenden Kollegen, meist mit dem Ziel, bei dem zu Begutachtenden keine Arbeitsunfähigkeit festzustellen.

Das Urteil des Bundesgerichts vom 13.06.2013 verunmöglicht die oben dargestellte Strategie. Dass sich manche betreuenden Ärzte bei der Begutachtung ihrer Patienten oft nicht entsprechend einbezogen fühlen, bleibt davon unberührt.

\section{Was kann man daraus lernen?}

Manche Beschwerdebilder bleiben nicht so eindeutig zuordenbar und Gutachter sollten am Beispiel CRF lernen, nicht vorschnell mit «keine organische Ursache keine Rente» zu begutachten. Das BGE-Urteil 9C_492/ 2014 vom 03.06.2015 geht in diese Richtung. Nicht die Diagnose, sondern der Nachweis der Behinderung soll mit Hilfe von Indikatoren im Fokus der Begutachtung stehen. Ob dieser Paradigmenwechsel in der Praxis auch inhaltlich vollzogen wird, ist fraglich [1].

Zusätzlich beachtenswert für die Beurteilung des CRF und ähnlicher Beschwerdebilder ist aus meiner Erfahrung:

- Verwendung der Diagnosekriterien für CRF [12], (s. Tabelle 1), auch wenn es keine anerkannte ICD-10 Diagnose ist.

- Einholen von Informationen vom Hausarzt und/ oder Onkologen.

- Einschätzung der funktionellen Einschränkungen mit Auswirkungen auf Aktivität und Partizipation.

- Die Prognose der CRF ist im Einzelfall kaum vorhersehbar, deshalb soll die Arbeitsunfähigkeit nach einer gewissen Zeit in der Regel reevaluiert werden.

- Das Bundesgerichtsurteil zu CRF wird bekannter werden. Es besteht die Möglichkeit - wie bei anderen Leiden auch - von vermehrter Aggravation oder Simulation, da objektive Parameter fehlen.

Daneben scheint mir eine kritische Reflexion der eigenen Haltung des Gutachters wichtig:

- Wie gehe ich mit Symptomen von zu Begutachtenden um, die ich nicht eindeutig somatisch begründet einordnen kann?

- Bin ich auf neue Literatur zu CRF neugierig, oder habe ich eine feste Meinung dazu?

- Überzeugt mich das Bundesgerichtsurteil über Cancer-related Fatigue?

- Überzeugen mich die Kriterien für das CRF?

- Gibt es für mich andere Kriterien, um die Diagnose eines Cancer-related Fatigue zu stellen?

- Denke ich über meine Vorurteile nach, die ich gegen Menschen habe, die ich begutachte? (Eigene Stereotypen gefährden eine professionelle Begutachtung [13].)
Alexander Kis Universitätsspital Basel Abt. Psychosomatik Bereich Medizin Hebelstrasse 2

CH-4031 Basel alexander.kiss[at]usb.ch 